\title{
Effects of the net charge on abundance and stability of supramolecular surfactant aggregates in gas phase
}

\author{
David Bongiorno, a,b Leopoldo Ceraulo, a,b* Gianluca Giorgi, ${ }^{\mathrm{c}}$ \\ Serena Indelicato, ${ }^{a, b}$ Mirella Ferrugia, ${ }^{a}$ Angela Ruggirello ${ }^{d}$ \\ and Vincenzo Turco Liveri $^{\mathrm{d}}$
}

Self-assembling of amphiphilic molecules under electrospray ionization (ESI) conditions is characterized by quite unexpected phenomenology. The noticeable differences with respect to the condensed phase are attributable to the absence of the surfactant-solvent interactions, the presence of net charge in the aggregates, and the strong deviation from equilibrium conditions. Aiming to investigate the effects of the net charge on abundance and stability of supramolecular surfactant aggregates, positively and negatively charged aggregates of sodium bis(2-ethylhexyl)sulfosuccinate (AOT) and sodium methane sulfonate (MetS), butane sulfonate (ButS) and octane sulfonate (OctS) have been studied by ESI mass spectrometry, energy resolved mass spectrometry and density functional theory calculations. The negatively charged aggregates are found to be less stable than their positive counterparts. The results are consistent with a self-assembling pattern dominated by electrostatic interactions involving the counterions and head groups of the investigated amphiphilic compounds while the alkyl chains point outwards, protecting the aggregates from unlimited growth processes. Copyright (c) 2011 John Wiley \& Sons, Ltd.

Keywords: self-assembling; anhydrous reverse micelles; electrospray ionization; energy-resolved mass spectrometry; DFT calculations

\section{Introduction}

Our interest in surfactant self-assembling in the gas phase led us to study in detail the structural and energetic features of positively and negatively charged aggregates of a typical surfactant molecule, ${ }^{[1,2]}$ sodium bis-(2-ethylhexyl)sulfosuccinate (AOT, Fig. 1), by electrospray ionization mass spectrometry (ESIMS), energy resolved mass spectrometry ${ }^{[3,4]}$ (ER-MS) and density functional theory (DFT) calculations.

Overall, these investigations support the hypothesis that, as a consequence of anisotropic surfactant-surfactant molecular interactions, AOT molecules self-assemble, forming a variety of either positively $\left[\mathrm{AOT}_{n}+\mathrm{Na}\right]^{+}$or negatively $\left[\mathrm{AOT}_{n}-\mathrm{Na}\right]^{-}$ monocharged aggregates that survive on the MS time scale. Independent of the mechanism of their formation (i) aggregation in the plasma state during droplet fission; (ii) aggregation into the droplets due to fast solvent evaporation; (iii) the decomposition of bigger aggregates; (iv) a mix of these mechanisms; these aggregates seem to be characterized by an anhydrous reverse micelle-like structure. Specifically, these structures are constituted by an internal hydrophilic core formed by the head groups of AOT and the corresponding counterions surrounded by their alkyl chains. Thus, such aggregation involves the formation under ESI conditions of charged supramolecular aggregates which appear from the outside as hydrophobic objects.

Besides, the observation that ionic clusters can be confined in the hydrophilic cores of these aggregates ${ }^{[2]}$ could also be of technological importance. In fact, it could be exploited in novel applications of surfactant aggregates in the gas phase, for instance as specialized air cleaning agents or carriers of pulmonary drugs.
In order to ascertain if the behavior shown by AOT is of general validity or system specific, recently we extended our investigations ${ }^{[4]}$ to sodium methane sulfonate (MetS), butane sulfonate (ButS) and octane sulfonate (OctS) (Fig. 1), i.e. amphiphilic molecules characterized by a smaller polar head (the sodium sulfonate group without the succinic ester moiety) linked to only one alkyl chain with a carbon atom number in the range 1-8.

In the gas phase, it was found that their cations show an aggregation pattern similar to that of AOT with minor effects attributable to the alkyl chain steric hindrance or the head group size. This finding appears particularly striking if one considers that, due to their very low solubility, MetS and ButS form neither direct micelles in aqueous solution ${ }^{[5]}$ nor reverse micelles in apolar media. Moreover, it underlines that the self-assembling of amphiphilic molecules in the gas phase constitutes a field distinct from that in the condensed phase, and it is characterized by a different

\footnotetext{
Correspondence to: Leopoldo Ceraulo, Centro Grandi ApparecchiatureUniNetLab, Università degli Studi di Palermo, Via Marini 14, I-90128 Palermo, Italy.E-mail:Iceraulo@unipa.it

a Dipartimento di Chimica e Tecnologie Farmaceutiche, Università degli Studi di Palermo, Via Archirafi 32, I-90123 Palermo, Italy

b Centro Grandi Apparecchiature-UniNetLab, Università degli Studi di Palermo, Via Marini 14, I-90128 Palermo, Italy

c Dipartimento di Chimica, Università degli Studi di Siena, Via Aldo Moro, I-53100 Siena, Italy

d DipartimentodiChimica-Fisica "F.Accascina", Università degliStudidiPalermo, Viale delle Scienze, I-90128 Palermo, Italy
} 


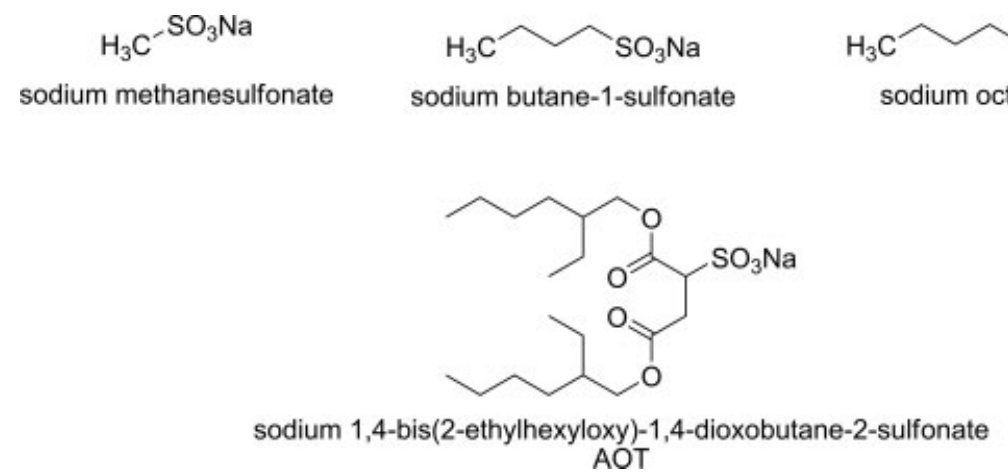

Figure 1. Structures of sodium methane sulfonate (MetS), butane sulfonate (ButS), octane sulfonate (OctS) and sodium bis(2-ethylhexyl)sulfosuccinate (AOT).

and sometimes unexpected phenomenology. The absence of the surfactant-solvent interactions, the presence of net charge in the aggregates and strong departures of the aggregate populations from equilibrium conditions could be the most prominent factors determining the observed peculiarities.

In order to enhance our knowledge in this novel research field and, in particular, to study the effect of the nature of the net charge on their abundances and stabilities, we have performed ESI-MS, MS/MS and energy-resolved MS experiments in the negative ion mode on the aggregates formed by MetS, ButS, OctS and AOT.

By comparing these data with those previously reported ${ }^{[1-4]}$ in positive mode, we will show that the abundances of negatively and positively charged aggregates are markedly different, and that the negatively charged aggregates of MetS, ButS and OctS are generally less stable than their positive counterparts. These findings and their interpretation will also be corroborated by the results of DFT calculations.

\section{Experimental Section}

Solvents used were LC-MS grade from Sigma-Aldrich (Germany).

Sodium salts of methanesulfonic acid (MetS, $\geq 98 \%$ ), butanesulfonic acid (ButS, $\geq 95 \%$ ), 1-octanesulfonic acid (OctS, $\geq 98 \%$ ) and of bis-(2-ethylhexyl)sulfosuccinate (AOT, $\geq 99 \%$ ) were purchased from Sigma-Aldrich (Germany).

ESI-MS and ESI-MS/MS spectra were made on a quadrupole time-of-flight high-resolution LC/MS system (Waters q-Tof premier) under the following experimental conditions: direct infusion at $10 \mu \mathrm{min}^{-1}$ flow rate, sample concentration $0.5 \mathrm{mM}$ (water/methanol, $1: 1$ ), i.e. the same as used for the experiment in positive mode, ${ }^{[4]}$ needle voltage $3000 \mathrm{~V}$, sampling cone $170 \mathrm{~V}$, extraction cone $4.4 \mathrm{~V}$, ion guide $1.2 \mathrm{~V}$, source temperature $90^{\circ} \mathrm{C}$, mass range: $50-8000 \mathrm{~m} / \mathrm{z}$, CID gas argon, desolvation temperature $250{ }^{\circ} \mathrm{C}$, desolvation gas $530 \mathrm{I} \mathrm{h}^{-1}$, scan time 1 or $2 \mathrm{~s}$, RF settings: autoprofile.

This experimental set up (sampling cone voltage $170 \mathrm{~V}$ ) could be considered as 'hard' ESI conditions. In fact, any solvated metal ions $\left[\left(\mathrm{CH}_{3} \mathrm{OH}\right)_{n} \mathrm{Na}\right]^{+}$were not detectable at this voltage. These species are observed at a lower cone voltage and disappear at voltages higher than $30 \mathrm{~V}$.

The intensity of each species in MS spectra has been calculated by the integral of the full isotopic pattern in order to account for the different isotopic abundances.
MS/MS spectra were obtained by setting the collision energy in order to reduce the precursor ion abundance to $50 \%$ of total ion current and averaging 11 consecutive scans.

The breakdown curves were obtained by changing the collision energy, by discrete values ranging from 2 to $70 \mathrm{~V}$. For each collision energy, an acquisition time of 1 min was set.

$\mathrm{KE}_{\mathrm{lab} 50 \%}$ of monocharged negative aggregates were averaged values of at least three independent experiments. Measured relative standard deviations per cent are always $\leq 10 \%$.

Geometry optimizations and energy calculations, corrected from the BSSE by using the counterpoise method, ${ }^{[6,7]}$ were performed by using the generalized gradient functional BP86 $6^{[8,9]}$ along with the resolution of identity approximation (RI-DFT) ${ }^{[10,11]}$ and the split valence plus polarization DZVP all-electron basis set of Godbout etal. (Basis sets were obtained from the Extensible Computational Chemistry Environment Basis Set Database, Version 02/02/06, as developed and distributed by the Molecular Science Computing Facility, Environmental and Molecular Sciences Laboratory which is part of the Pacific Northwest Laboratory, Richland, Washington, USA, and funded by the U.S. Department of Energy. ${ }^{[12]}$ This approach provides a sufficiently detailed and accurate method to gain structural and energetic information on aggregates of surfactant anions and alkali cations. ${ }^{[3,4]}$ All calculations were performed by using the Gaussian 03 package. ${ }^{[13]}$

\section{Results and Discussion}

In our experimental conditions, analogously to the gas-phase behavior of cationic MetS, ButS and OctS aggregates, ${ }^{[4]}$ those negatively charged also show mainly singly charged species, such as $\left[\mathrm{MetS}_{n}-\mathrm{Na}^{+}\right]^{-},\left[\mathrm{ButS}_{n}-\mathrm{Na}^{+}\right]^{-}$and $\left[\mathrm{OctS}{ }_{n}-\mathrm{Na}^{+}\right]^{-}$ions with $n$ up to 25 , and solvated species are not observed.

Concerning the abundances of these ionic clusters, a direct comparison of the absolute values between positively and negatively charged species is not feasible due to the intrinsic different ion current between ESI spectra obtained in positive and negative ion modes. Further, an 'indifferent marker' as internal reference cannot be used, because it could be entrapped in the surfactant clusters, giving mixed aggregates.

Thus, as a compromise between the need for additional information and attainable data, the abundances of each aggregate in positive and negative mode (Fig. 2 on the right) were further normalized by dividing by the charged monomer abundance (Fig. 2 on the left). 

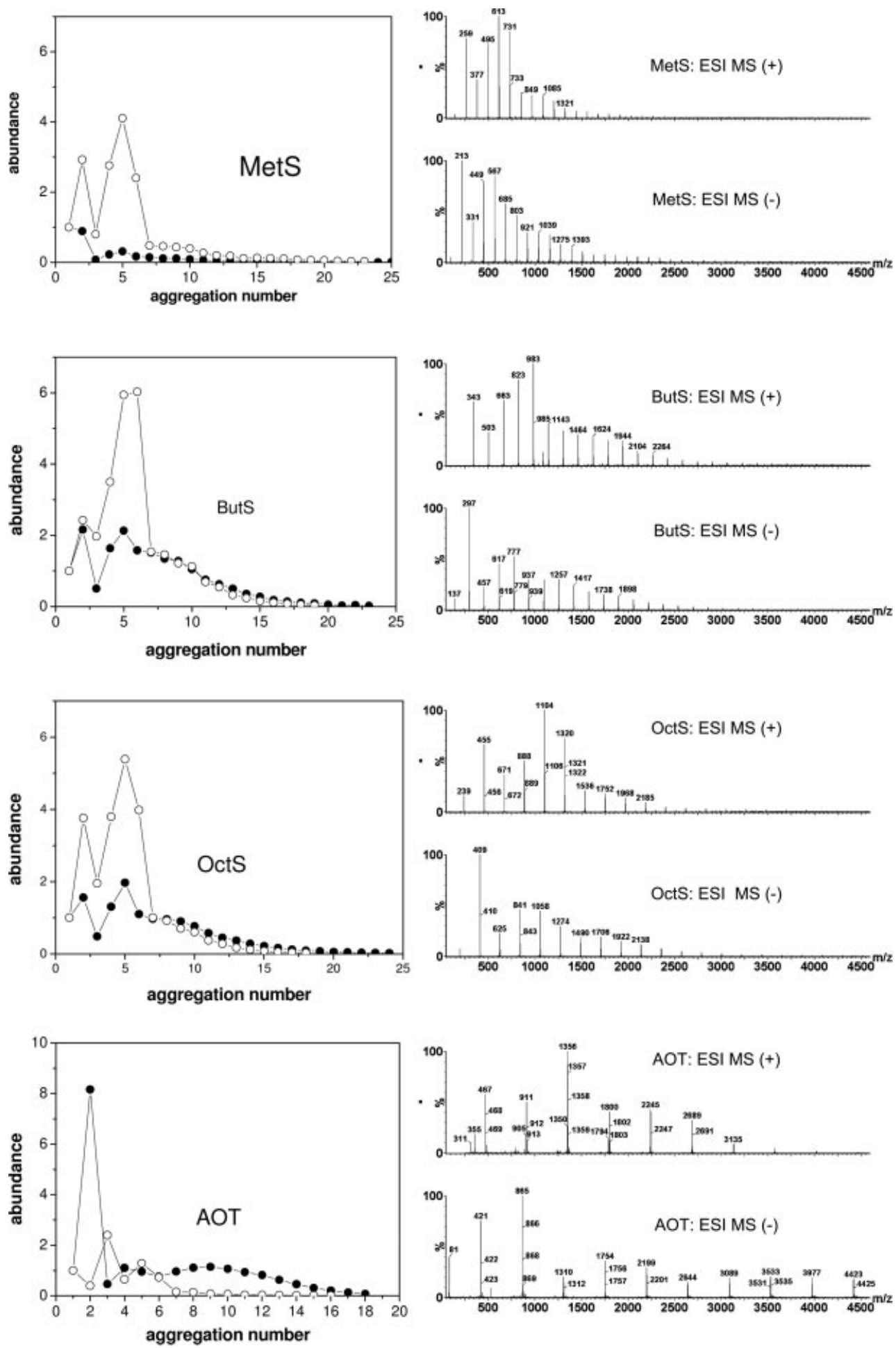

Figure 2. On the left are reported the normalized abundances as a function of the aggregation number for positively (circles) and negatively (black dot) charged MetS, ButS, OctS and AOT aggregates. On the right the corresponding ESI MS spectra.

Some striking differences emerge from a comparative analysis of these data:

(1) marked differences in the normalized abundances between positively negatively charged aggregates, and especially at the low aggregation number $(n)$, the occurrence of aggregates showing an extra abundances;
(2) the positively charged aggregates at low $n$ of MetS, ButS and OctS show relative abundance higher than the negatively charged ones;

(3) ButS and OctS show relative abundances higher than MetS both at the low and high $n$ values;

(4) the profile of aggregates produced by AOT is significantly different from those of the sodium alkyl sulfonates; 
(5) the abundance profiles are characterized by the superposition of a narrow distribution centered at low $n$ with a broad one centered at large $n$. This is particularly evident in the sulfonates case where cations tend to cluster better than anions while the opposite occurs for AOT.

All these features can be taken as clues of the structural and energetic properties regarding the charged aggregates and of the processes controlling their formation/decomposition. In particular, the predominance of singly charged aggregates and the different profiles of positively and negatively charged species indicate that the net charge location within the aggregates should influence significantly their occurrence. According to molecular dynamic simulations, ${ }^{[14]}$ this suggests a self-assembling pattern mainly driven by the strong electrostatic interactions among the positive counterions and negative head groups of the amphiphilic compounds, thus forming a hydrophilic core surrounded by their alkyl chains. It is worth noting that steric reasons impose an asymmetric location of the positive counterions and negative head groups within the anhydrous reverse micelle-like aggregates.

In agreement with this picture, the absence of solvated species can be explained by assuming that the solvent-amphiphile interactions are much weaker than amphiphile-amphiphile ones and it suggests that the energies involved during droplet fragmentation are able to totally desolvate amphiphilic molecules.

Moreover, the higher abundances of ButS and OctS aggregates with respect to those produced by MetS (Fig. 2) unveil the protective role of a long alkyl chain toward unlimited cluster coalescence and separation from the gas phase.

It is worth noting that the normalized abundances of negatively monocharged aggregates produced by AOT are generally higher than those of positively charged ones. Furthermore, while MetS, ButS and OctS show almost the same distribution of the most abundant species irrespective of the overall charge, AOT has a different behavior. While its negative species are dominated by the dimer $\left[\mathrm{AOT}_{2}-\mathrm{Na}^{+}\right]^{-}$, the most abundant positive ion is the trimer $\left[\mathrm{AOT}_{3}+\mathrm{Na}^{+}\right]^{+}$(Fig. 2).

Keeping in mind that aggregation is driven by ionic interactions and that in both negative and positive ion modes the growth of the aggregates is due to sodium bridge formation between sulfonate groups of the polar heads of surfactant, the behavior of AOT should be due to the very high steric hindrance of its two branched alkyl chains that shield the polar core of $\left[\mathrm{AOT}_{2}-\mathrm{Na}^{+}\right]^{-}$ as well as that of $\left[\mathrm{AOT}_{3}+\mathrm{Na}^{+}\right]^{+}$by further polar interactions.

DFT calculations aimed at obtaining information about structure of aggregates were carried out. Some representative optimized structures of negatively charged surfactant aggregates are shown in Fig. 3. It should be noted that sodium counterions and hydrophilic head groups are in the center of the aggregates while the surfactant alkyl chains are directed outwards. This spatial organization indicates that triggered by the steric constraints imposed by alkyl chains, electrostatic interactions are the gluing agent responsible for the formation and stability of aggregates.

Analogous to the previous work on positively charged ions, ${ }^{[4]} \mathrm{MS} / \mathrm{MS}$ experiments have been carried out by selecting the peaks corresponding to $\left[\mathrm{MetS}_{n}-\mathrm{Na}^{+}\right]^{-}$, $\left[\mathrm{ButS}_{n}-\mathrm{Na}^{+}\right]^{-}$and $\left[\mathrm{OctS}_{n}-\mathrm{Na}^{+}\right]^{-}(n=2,3,5,7,10)$ aggregates as precursor ions. For a comparison, the same experiments were performed on the $\left[\mathrm{AOT}_{n}-\mathrm{Na}^{+}\right]^{-}$aggregate. The MS/MS spectra of the pentamer species are shown in Fig. $4(\mathrm{a}-\mathrm{d})$ as a representative example.

The MS/MS spectra of $\left[\mathrm{MetS}_{n}-\mathrm{Na}^{+}\right]^{-},\left[\mathrm{ButS}_{n}-\mathrm{Na}^{+}\right]^{-}$and $\left[\mathrm{OctS} \mathrm{S}_{n}-\mathrm{Na}^{+}\right]^{-}$as well as those of $\left[\mathrm{AOT}_{n}-\mathrm{Na}^{+}\right]^{-}$are very simple and characterized by formal losses of (AlkS) $m(1 \leq m \leq(n-1))$ as neutral species, that could be due to sequential losses of monomer and/or to multimers ejection. Fragmentation of the alkyl chains is not observed, excepted for AOT that, at high collision energies, loses in small amounts octene (112 Da) through a hydrogen rearrangement involving a carbonyl group. ${ }^{[2,4]}$

Concerning aggregates stability, theoretical calculations reported in our previous papers ${ }^{[1-3,14]}$ indicated that negative AOT aggregates are less stable than corresponding positive ones. This can be easily rationalized considering that the presence of an extra sodium ion involves additional ionic bonds and consequently an increased stability. However, no experimental evidence has been reported yet to support this hypothesis.

In order to obtain such information, ER-MS experiments have been performed. They consist of obtaining tandem mass spectra as a function of laboratory collision energy, thus allowing determining and comparing the stability of different precursor ions, the energy of their decompositions, and the relative abundance of their product ions (the branching ratio).

The breakdown curves of 2-, 3-, 5-, 7- and 10-mer aggregates of MetS, ButS, OctS and AOT have been determined. Those of pentamers are reported in Fig. $5(\mathrm{a}-\mathrm{d})$ as a representative example.

As expected, the breakdown curves of the precursor ions shift at increasing laboratory kinetic energy $\left(\mathrm{KE}_{\mathrm{lab}}\right)$, increasing the surfactant chain size (Fig.5) or the aggregation number (not shown).

Information on the relative stability of the monocharged aggregates are better obtained by kinetic energy in the centerof-mass frame of reference $\left(\mathrm{KE}_{\mathrm{com}}\right)$ associated with a $50 \%$ survival yield of the parent ions $\left(\mathrm{KE}_{\mathrm{com} 50 \%}\right)$ that have been determined from the laboratory kinetic energy required to dissociate $50 \%$ of the selected species $\left(\mathrm{KE}_{\text {lab50\% }}\right)$, by the equation

$$
\mathrm{KE}_{\mathrm{com} 50 \%}=\mathrm{KE}_{\mathrm{lab} 50 \%} \times m_{n} /\left(m_{n}+m_{i}\right)
$$

where $m_{n}$ is the mass of the neutral target gas and $m_{i}$ the mass of the ion of interest. ${ }^{[15-19]}$ This quantity corresponds to the maximum of internal energy that could be transferred to the aggregate, in the case of one totally inelastic binary collision with the target gas, when $50 \%$ of the precursor ion dissociates. This condition is generally not true: in fact, the fraction of $\mathrm{KE}_{\mathrm{com} 50 \%}$ which is converted in internal energy of the aggregate depends on several factors such as collision details and aggregate structure, multiple random collisions and finally, on aggregate cross section.

The $\mathrm{KE}_{\mathrm{com} 50 \%}$ values, calculated according to $\mathrm{Eq}$ (1) as a function of the aggregation number, are shown in Fig. 6. Moreover, the calculated energy changes accompanying the loss of surfactant monomer from surfactant charged aggregates up to $n=7$ for alkyl sulfonates have been also added in the insets. In the AOT case, due to the huge increase in the computational time with aggregate atom numbers, calculations were only performed up to $n=4$.

It can be noted that $\mathrm{KE}_{\mathrm{com} 50 \%}$ and calculated energy changes show qualitatively similar trends meaning that experimental measurements mainly reflect the aggregates stability. Moreover, this finding is consistent with an anhydrous reverse micelle-like structure of surfactant aggregates in gas phase.

The greater stability of positively charged aggregates with respect to the negatively charged ones is definitively experimentally proven. In particular, it can be noted that the highest $\mathrm{KE}_{\mathrm{com} 50 \%}$ of the sulfonates and AOT in negative mode is shown by the 2-mers that agree with the very large predominance of the corresponding 
(a)

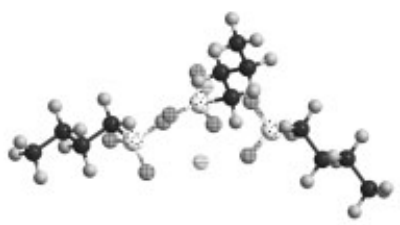

(c)

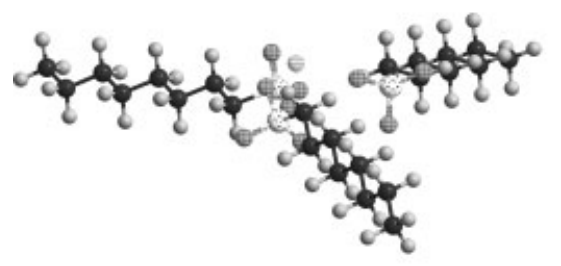

(b)

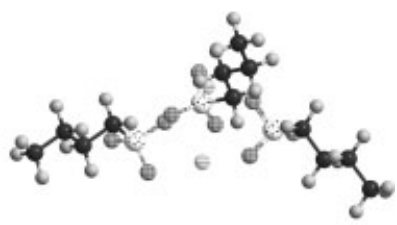

(d)

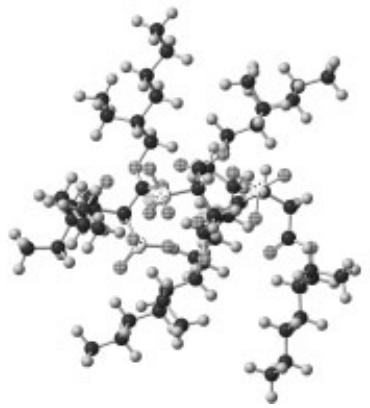

Figure 3. Optimized geometries of negatively charged 3-mers of MetS (a), ButS (b), OctS (c) and AOT (d). H, Light gray; C, gray; S, dotted white; O, squares pattern; $\mathrm{Na}$, lines pattern.
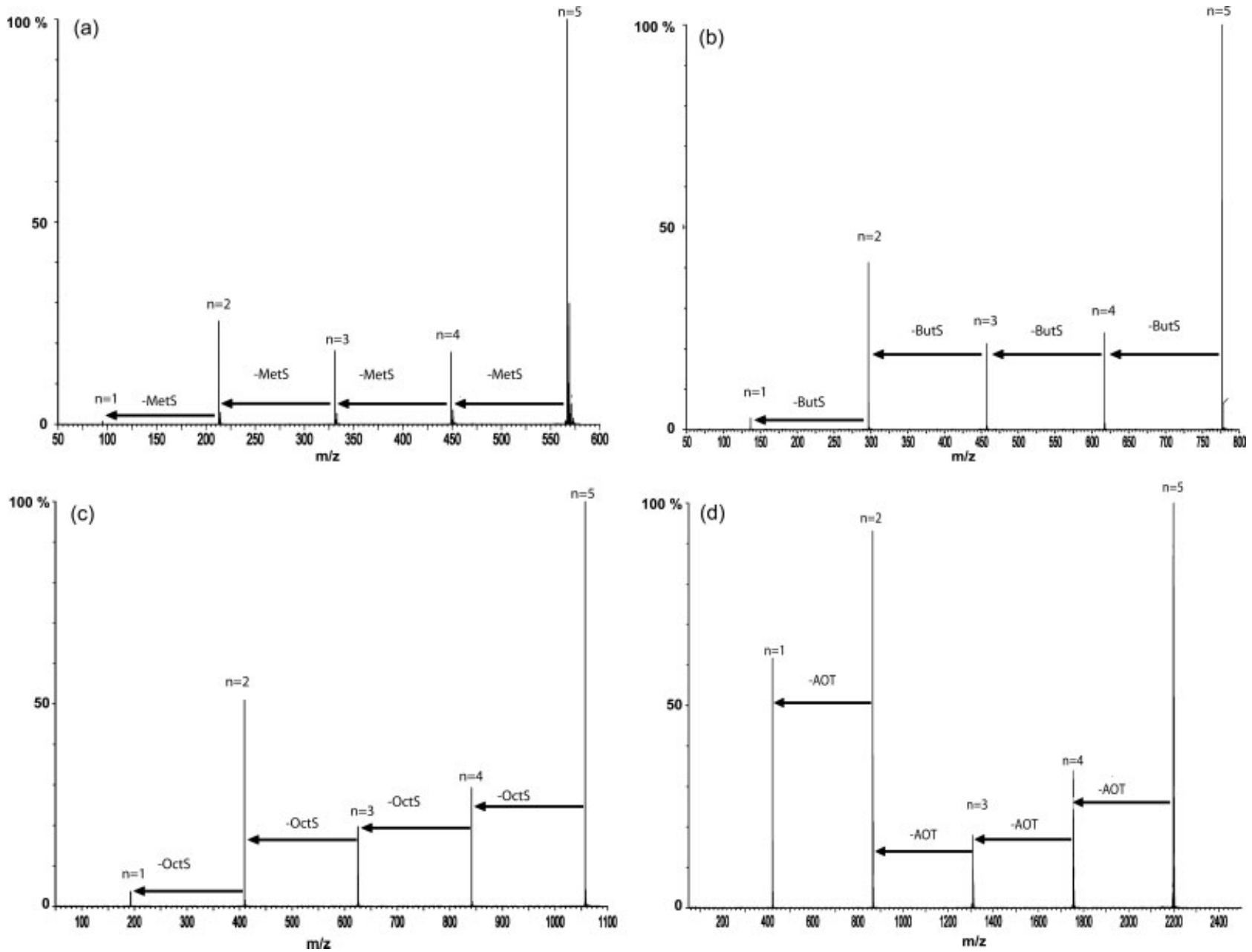

Figure 4. (a-d) ESI-MS/MS spectra (negative mode) of 5-mer species: (a) MetS; (b) ButS; (c) OctS and (d) AOT. 

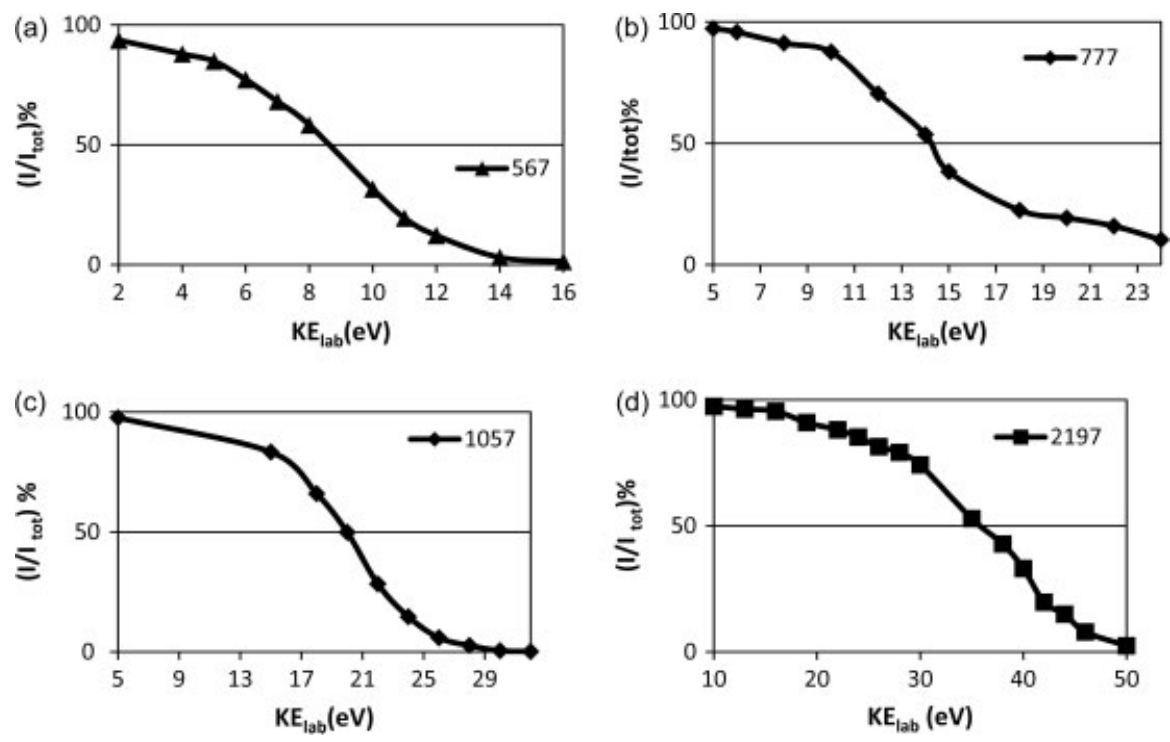

Figure 5. (a-d) Breakdown curves of negatively charged 5-mers: (a) MetS; (b) ButS; (c) OctS and (d) AOT.
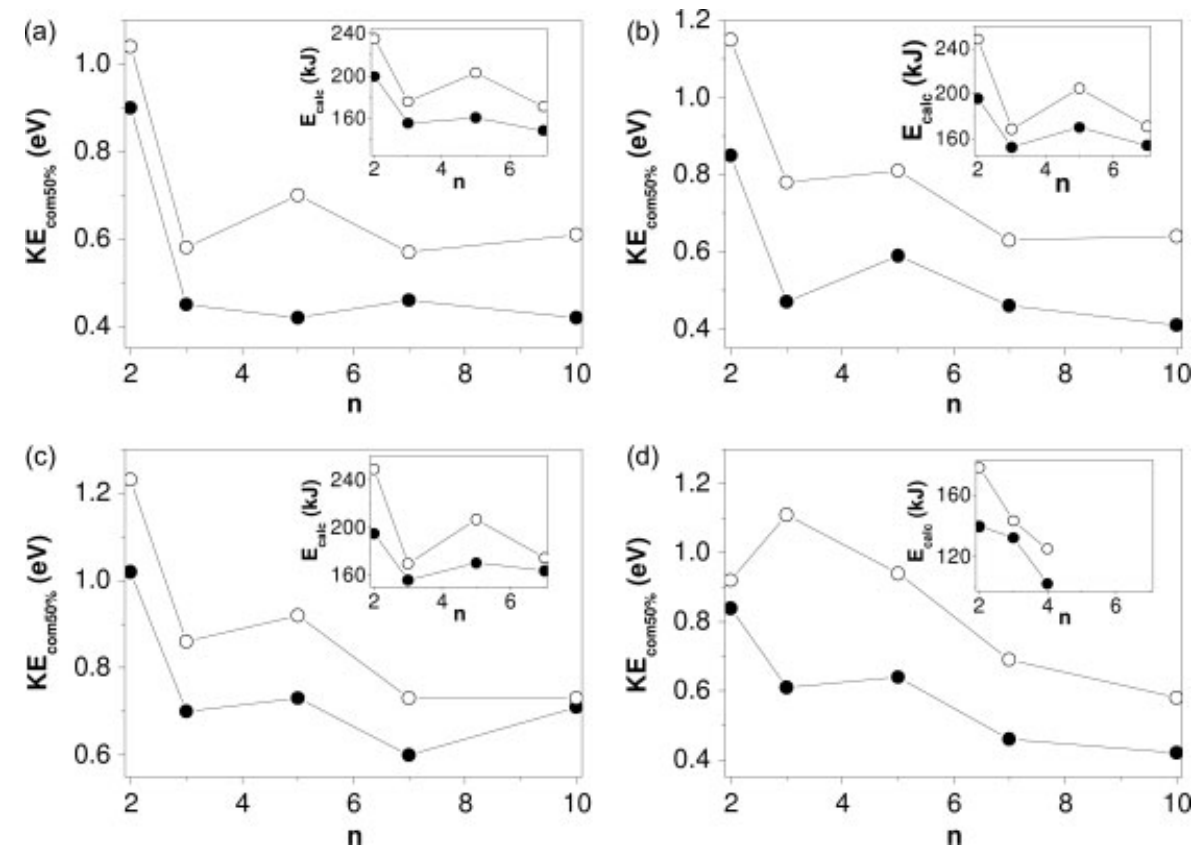

Figure 6. $\mathrm{KE}_{\mathrm{com} 50 \%}(\mathrm{eV})$ of positively (circle) and negatively (black dot) monocharged aggregates of MetS (a), ButS (b), OctS (c) and AOT (d) as a function of the aggregation number $(n)$. The calculated energy changes accompanying the loss of surfactant monomer from charged aggregates are shown in the insets.

peak in the ESI-MS spectra of all the examined compounds. Similarly, an extra stability of the pentamer is observed for negative clusters of ButS, OctS and AOT. At higher $n$ values $\mathrm{KE}_{\text {com } 50 \%}$ slowly decreases, tending to converge to a constant value that is between 0.4 and $0.7 \mathrm{eV}$.

\section{Conclusions}

Analogous to what previously observed in the case of their cationic aggregates, the electrospray ionization mass spectra of negatively charged species of sodium MetS, ButS and OctS and of AOT demonstrated the presence of monocharged supramolecular aggregates with an aggregation number up to 25 .
Solvated species are undetectable, thus evidencing that, as observed for cations, in negatively charged aggregates the interactions between amphiphilic molecules are also stronger than those with the solvent.

Significant differences in the abundances of positively and negatively charged aggregates, attributable to the different location of the charges within the anhydrous reverse micelle-like aggregate core, have been observed.

ER-MS, together with DFT calculations allowed to rationalize the occurrence of species showing an extra abundance, even if kinetic aspects are also to be considered. Due to the alkyl chains pointing outwards and protecting the aggregates from an unlimited growth process, the growth limitation seems to be also related to the steric 
hindrance of the surfactant apolar moiety. Apart from the species showing an extra abundance, the stability of the aggregates, estimated through the center-of-mass collision energy required to dissociate $50 \%$ of the population, mildly depends on the length of the alkyl chain and the aggregation number while the presence of carbonyl groups in AOT do not introduces significant differences with respect to alkanesulfonates behavior.

Further, ER-MS gives experimental evidence that the negatively charged aggregates are less stable than their positive counterparts with the same aggregation number.

Overall, experimental findings are consistent with a selfassembling pattern dominated by the counterion and head group electrostatic interactions of the investigated amphiphilic compounds while DFT calculations agree with an anhydrous reverse micelle-like aggregation model.

\section{Acknowledgements}

The support of University of Palermo (Fondi di Ricerca Scientifica ex $60 \%$ ) is gratefully acknowledged. Mass spectrometry experimental data were provided by Centro Grandi Apparecchiature, UniNetLab, Università di Palermo funded by P.O.R. Sicilia 2000-2006, Misura 3.15 Quota Regionale.

\section{References}

[1] D. Bongiorno, L. Ceraulo, A. Ruggirello, V. Turco Liveri, E. Basso, R. Seraglia, P.Traldi. Surfactant self-assembling in gas phase: electrospray ionization and matrix-assisted laser desorption/ionization-mass spectrometry of singly charged AOT clusters. J. Mass Spectrom. 2005, 40, 1618

[2] G. Giorgi, L. Ceraulo, V. Turco Liveri. Surfactant self-assembly in the gas phase: bis(2-ethylhexyl)sulfosuccinate-alkaline metal ion aggregates. J. Phys. Chem. B 2008, 112, 1376.

[3] G. Giorgi, E. Giocaliere, L. Ceraulo, A. Ruggirello, V. Turco Liveri. Spatially ordered surfactant assemblies in the gas phase: negatively charged bis(2-ethylhexyl)sulfosuccinate-alkaline metal ion aggregates. Rapid Commun. Mass Spectrom. 2009, 23, 2206.

[4] D. Bongiorno, L. Ceraulo, G. Giorgi, S. Indelicato, A. Ruggirello, V. Turco Liveri. Supramolecular aggregates in vacuum: positively monocharged sodium alkanesulfonate clusters. Eur. J. Mass Spectrom. 2010, 16, 151.

[5] M. G. Khaledi. Micelles as separation media in high-performance liquid chromatography and high performance capillary electrophoresis: overview and perspective. J. Chromatogr. A 1997, $780,3$.

[6] S. F. Boys, F. Bernardi. The calculation of small molecular interactions by the differences of separate total energies. Some procedures with reduced errors. Mol. Phys. 1970, 19, 553.
[7] S.Simon, M.Duran, J. J.Dannenberg. How does basis set superposition error change the potential surfaces for hydrogen bonded dimers? J. Chem. Phys. 1996, 105, 11024.

[8] A.D. Becke. Density-functional exchange-energy approximation with correct asymptotic behavior. Phys. Rev. A 1988, 38, 3098.

[9] J.P. Perdew. Density-functional approximation for the correlation energy of the inhomogeneous electron gas. Phys. Rev. B 1986, 33, 8822.

[10] K. Eichkorn, O. Treutler, H. Öhm, M. Häser, R. Ahlrichs. Auxiliary basis sets for main row atoms and transition metals and their use to approximate coulomb potentials. Chem. Phys. Lett. 1995, 240, 283.

[11] K. Eichkorn, F. Weigend, O. Treutler, R. Ahlrichs. Auxiliary basis sets for main row atoms and transition metals and their use to approximate coulomb potentials. Theor. Chem. Acc. 1997, 97, 112.

[12] N. Godbout, D. R. Salahub, J. Andzelm, E. Wimmer. Optimization of gaussian-type basis sets for local spin density functional calculations. Part I. Boron through neon, optimization technique and validation. Can. J. Chem. 1992, 70, 560.

[13] M. J. Frisch, G. W. Trucks, H. B. Schlegel, G. E. Scuseria, M. A. Robb, J.R. Cheeseman, J.A. Montgomery Jr., T. Vreven, K. N. Kudin, J.C. Burant, J.M. Millam, S. S. lyengar, J.Tomasi, V. Barone, B. Mennucci, M. Cossi, G. Scalmani, N. Rega, G. A. Petersson, H. Nakatsuji, M. Hada, M. Ehara, K. Toyota, R. Fukuda, J. Hasegawa, M. Ishida, T. Nakajima, Y. Honda, O. Kitao, H. Nakai, M. Klene, X. Li, J. E. Knox, H.P. Hratchian, J.B. Cross, V. Bakken, C. Adamo, J. Jaramillo, R. Gomperts, R. E. Stratmann, O. Yazyev, A. J. Austin, R. Cammi, C. Pomelli, J. W. Ochterski, P. Y. Ayala, K. Morokuma, G. A. Voth, P. Salvador, J. J. Dannenberg, V. G. Zakrzewski, S. Dapprich, A.D.Daniels, M.C.Strain, O. Farkas, D. K. Malick, A. D. Rabuck， K. Raghavachari, J. B. Foresman, J. V. Ortiz, Q.Cui, A. G. Baboul, S.Clifford, J.Cioslowski, B. B. Stefanov, G. Liu, A. Liashenko, P. Piskorz, I. Komaromi, R. L. Martin, D. J. Fox, T. Keith, M. A. Al-Laham, C.Y.Peng, A. Nanayakkara, M. Challacombe, P.M. W. Gill, B. Johnson, W. Chen, M. W. Wong, C. Gonzalez, J. A. Pople. Gaussian 03, Revision D.02, Gaussian, Inc., Wallingford, $\mathrm{CT}, 2004$.

[14] G. Longhi, S. L. Fornili, V. Turco Liveri, S. Abbate, D. Rebeccani, L. Ceraulo, F. Gangemi, Sodium bis(2-ethylhexyl)sulfosuccinate selfaggregation in vacuo: molecular dynamics simulation. Phys. Chem. Chem. Phys. 2010, 12, 4694.

[15] L. L.Griffin, D. J.McAdoo. The effect of ion size on rate of dissociation: RRKM calculations on model large polypeptide ions. J. Am. Soc. Mass Spectrom. 1993, 4, 11.

[16] V. Gabelica, E. De Pauw. Internal energy and fragmentation of ions produced in electrospray sources. Mass Spectrom. Rev. 2005, 24, 566.

[17] A. K. Shulka, J. H. Futrell. Collisional activation and dissociation of polyatomic ions. Mass Spectrom. Rev. 1993, 12, 211.

[18] S. A. McLuckey. Principles of collisional activation in analytical mass spectrometry. J. Am. Soc. Mass Spectrom. 1991, 3, 599.

[19] A. K. Shulka, J. H. Futrell. Tandem mass spectrometry: dissociation of ions by collisional activation. J. Mass Spectrom. 2000, 35, 1069. 